TRANS · núm. $24 \cdot 2020$

MISCELÁNEA $\cdot 167-190$
La globalización de los mercados y el creciente intercambio comercial entre países han conllevado un aumento de las reclamaciones judiciales debido a problemas relacionados con el fraude. Esto ha hecho que la auditoría forense cobre especial relevancia en el ámbito judicial, así como los informes periciales contables que surgen de su actividad, que se han convertido por tanto en un encargo habitual en traducción jurídico-económica. El objetivo de este artículo es realizar una primera aproximación a la caracterización de un informe pericial contable a través de un estudio de corpus comparable (inglés-español) ad hoc, lo que nos permitirá detallar su macroestructura y microestructura y analizar las principales unidades terminológicas que se encuentran para esta combinación lingüística. Esto permitirá conocer un poco más en detalle las principales características de este tipo de documentos como paso previo a su traducción.

PALABRAS CLAVE: corpus, informe pericial contable, traducción jurídicoeconómica.

\title{
Primera aproximación a la caracterización de los informes periciales contables (inglés- español) en el ámbito judicial mediante un estudio de corpus
}

Elena Alcalde Peñalver

Universidad de Alcalá

AleXANDRa Santamaría Urbieta Universidad Internacional de La Rioja

\section{A First Approach to the Characterization of Accounting Forensic Reports (English-Spanish) in Legal Settings Through a Corpus-Based Methodology}

The globalization of markets and the increasing commercial exchange between countries has led to a rise in legal claims due to problems related to fraud. This has made forensic auditing particularly relevant in legal settings, as well as the accounting forensic reports that arise from its activity, which have thus become a regular task in legal-economic translation. For this reason, the aim of this paper is to provide an approach to the characterization of an accounting forensic report by using an ad hoc comparable corpus (EnglishSpanish), which will allow us to detail the macrostructure and microstructure of this text and analyze the main terminological units found for this language combination. This will provide specific details on the main characteristics of these documents as a previous step for their translation.

KEY WORDS: corpus, accounting forensic report, legal-economic translation 


\section{1. INTRODUCCIÓN}

En la actualidad, la traducción de informes periciales contables que se presentan en un juicio como medio de prueba se ha convertido en un encargo habitual en el campo de la traducción jurídico-económica. Esto se debe al aumento de las reclamaciones judiciales ante la globalización de los mercados, lo que incluso ha obligado a muchas empresas a contar con departamentos específicos dedicados a la actividad judicial (forensic departments) para la elaboración de estos informes, que sirven como prueba en procedimientos judiciales y arbitrales (Bermúdez, 2019). En efecto, en la primera década del siglo xxi se produjeron numerosos escándalos y fraudes corporativos que contribuyeron al desarrollo de una gran crisis económica y que puso en evidencia la creciente necesidad de la contabilidad forense (Ball, 2009), definida por Huber (2012: 259) como "the venue within which the cultural mediation of legal and economic claims is accomplished". En este ámbito confluyen tanto aspectos jurídicos como económicos con el objetivo de investigar posibles fraudes en el seno empresarial (Singleton y Singleton, 2010). De hecho, en EE. UU. los abogados son los que más requieren los servicios de los contables forenses (Davis, Farrell y Ogilby, 2010) y Stanbury y Paley-Menzies (2010:v) lo llegan a definir como una ciencia "of gathering and presenting information in a form that will be accepted by a court of jurisprudence against perpetrators of economic crime".

Si tenemos en cuenta los países en los que enmarcamos nuestro estudio (España y EE. UU.), el ámbito de la contabilidad forense cobra aún más relevancia debido a las importantes relaciones comerciales que se desarrollan entre ambos. En este sentido, es necesario señalar que, según los últimos datos publicados por la Secretaría de
Estado de Comercio (2020), EE. UU. es el principal socio comercial de nuestro país fuera de la Unión Europea: en 2019 este mercado supuso un $4,7 \%$ de las exportaciones españolas de bienes y en 2017 fue el primer inversor en términos de stock, así como el segundo destino de la inversión española.

Estos datos son una mínima representación de todo lo que suponen las relaciones económicas entre países a nivel mundial. Ante esta situación de continuos intercambios, y lo que conlleva operar en diferentes ámbitos jurisdiccionales y lingüísticos, resulta normal afirmar que las empresas hoy en día están expuestas a numerosos riesgos, entre los que se encuentran los relacionados con acciones fraudulentas. Esto queda patente en los numerosos casos de fraude corporativo que se presencian en la actualidad de forma habitual (Deloitte, 2019). Un ejemplo de ello lo demuestra el artículo publicado en 2019 en el periódico de economía Cinco Días, en el que se explicaban los indicios de fraude que había revelado el informe pericial contable fruto de la auditoría forense que el grupo de supermercados DIA había encargado para investigar sus cuentas. De forma específica, según indica el último informe publicado por la Asociación de Examinadores de Fraude Certificados (ACFE, por sus siglas en inglés) (2020), los casos de fraude suponen pérdidas de un promedio de $8.300 \$$ al mes a las empresas, lo que representa un $5 \%$ de las pérdidas anuales.

Estos datos demuestran la relevancia que tiene hoy en día la auditoría forense en el ámbito judicial y los informes periciales contables que surgen de su actividad. Sin embargo, no existen hasta la fecha estudios que se centren, desde el punto de vista traductológico, en la caracterización de estos documentos como paso previo a la traducción de este tipo de textos. Asimismo, es importante que la investigación en el ámbito de 
la traducción esté en consonancia con las exigencias del mercado profesional con el fin de ofrecer a los docentes material que les ayude a incluir en sus clases la traducción de estos textos. De esta forma, el objetivo de este artículo es realizar una primera aproximación a la caracterización de un informe pericial contable que se utiliza en el ámbito judicial a través de un estudio de corpus comparable (inglés-español) ad hoc, lo que nos permitirá detallar la macroestructura y microestructura de este texto y analizar las principales unidades terminológicas que se encuentran para esta combinación lingüística.

Para ello, en primer lugar, comenzaremos nuestro artículo con una contextualización de nuestro objeto de estudio para que se pueda conocer en qué consiste un informe pericial contable en el ámbito judicial. Seguidamente realizaremos una revisión de los estudios previos en el ámbito jurídico-económico que se centran en el análisis del género textual y de corpus como punto de partida para aportar recursos que faciliten la tarea del traductor, así como en su introducción en el aula de traducción. En la metodología detallaremos el procedimiento que hemos seguido para llevar a cabo nuestro estudio y en el apartado de análisis de resultados expondremos los datos obtenidos. Finalmente, en las conclusiones reflexionaremos sobre la importancia de este tipo de estudios para la profesión, investigación y docencia de la traducción en el ámbito jurídico-económico.

\section{MARCO TEÓRICO}

En este apartado realizaremos en primer lugar una contextualización de nuestro objeto de estudio en la que se definirá en qué consiste un informe pericial contable en el ámbito judicial. Asimismo, compararemos las partes de las que se supone que, según la teoría debe constar, tan- to en España como en EE. UU., lo que contrastaremos más adelante con los textos reales que formarán parte de nuestro corpus. Por último, analizaremos los estudios que tratan la hibridación textual en traducción jurídico-económica y los análisis de corpus que se han llevado a cabo en este ámbito.

\section{1 ¿Qué es un informe pericial contable en el ámbito judicial?}

Un «dictamen pericial» o un «informe pericial» contable en el ámbito judicial es un documento que resulta de la labor profesional y específica desarrollada por un experto contable sobre un aspecto económico determinado y que contribuye a su consideración como prueba en un procedimiento judicial (Pierino Dell'Elce, 2017: 4). Este tipo de informes se regula en el apartado 1 del art. 299 de la Ley de Enjuiciamiento Civil (LEC) de España. Este mismo autor añade que, cuando se requiere este tipo de pruebas para resolver un procedimiento judicial, es un contable experto el que debe analizar y examinar una serie de puntos dentro de un «cuestionario o pliego pericial» sobre los que deberá emitir un dictamen determinado.

En cuanto a las características del documento, Bermúdez (2019, p. 53-54) señala que estas son las básicas que el perito contable debe tener en cuenta a la hora de emitir su informe:

- Veracidad y objetividad. Esto quiere decir que el experto deberá emitir el informe en base a hechos determinados y mediante una metodología adecuada que permita que los resultados se ajusten a la realidad.

- Informe conciso y completo. Se deberán aportar todos los datos que sean necesarios para describir en la totalidad los hechos que se quieran probar, sin que esto implique que 
170 la descripción de los mismos se haga de una forma extensa que impida la comprensión de los mismos.

- Claridad e inteligibilidad. El lenguaje especializado que utilice el perito contable deberá ser comprensible y de acuerdo a las circunstancias de los destinatarios.

En cuanto a su estructura y contenido, es necesario analizarlo desde el punto de vista del derecho comparado para así poder observar si existen diferencias entre España y Estados Unidos. Por un lado, tal y como indica Bermúdez (2019), la LEC no contiene disposiciones que regulen la forma o contenido que debe tener el «dictamen» que emite el perito. Sin embargo, en el artículo 478 de la Ley de Enjuiciamiento Criminal (LECr) se hace referencia a las siguientes partes que deberá comprender, si fuera posible, el informe pericial:

I. Descripción de la persona o cosa que sea objeto del mismo en el estado o del modo en que se halle.

El secretario extenderá esta descripción, dictándola los peritos y suscribiéndola todos los concurrentes.

2. Relación detallada de todas las operaciones practicadas por los peritos y de su resultado, extendida y autorizada en la misma forma que la anterior.

3. Las conclusiones que en vista de tales datos formulen los peritos conforme a los principios y reglas de su ciencia o arte.

En relación a la estructura, Bermúdez (2019) señala que, a pesar de la falta de indicaciones en la legislación al respecto, es necesario que los informes periciales contables contengan las siguientes partes para favorecer su comprensión:

- Identificación y manifestaciones legales. En esta primera parte se aportarán los datos del tipo de documento que se trate (dicta- men en caso de jurisdicción civil, mercantil, laboral o contencioso-administrativa e informe en caso de penal). También aparecerá la indicación de a quién se dirige el informe (juzgado, localidad y número de procedimiento). Seguidamente, aparecerán los datos del perito, un índice del informe y las manifestaciones legales en las que el perito manifiesta bajo juramento o promesa decir la verdad y trabajar con total objetividad.

- Objeto del informe. En esta parte se indica la naturaleza y contenido del encargo.

- Documentación utilizada. Se relacionarán los documentos que han servido para obtener la evidencia a la que se hace referencia en el informe (cuentas anuales, libros de contabilidad, etc.).

- Párrafo de reserva de información. Indica que la evidencia que se ha obtenido es a reserva de otros antecedentes o hechos que puedan existir y que podrían hacer variar la situación expuesta en el informe y sus conclusiones.

- Antecedentes y contexto de la situación. Se indican las partes del proceso y se expone la razón por la que se ha pedido al perito que actúe.

- Procedimientos y metodología aplicados. Se exponen los medios y metodología utilizados para la realización del informe.

- Conclusiones. En esta parte aparecen de forma numerada las conclusiones obtenidas del trabajo realizado. En procedimientos penales siempre debe aparecer, pero en civiles, laborales y contencioso-administrativos se puede suprimir si el contenido ha quedado explícito con anterioridad.

- Cláusula de limitación de distribución. Se indica que se trata de un informe que se utilizará solamente para el proceso para el cual se haya solicitado. 
- Documentación anexa. Se adjuntan los documentos que haya utilizado el perito para la realización de su trabajo.

Por su parte, en EE. UU. son los juzgados de cada estado los que determinan si admiten o no admiten estos informes periciales como prueba (Huber, 2013). Estos peritos deben trabajar siguiendo las normas impuestas por la Comisión de Bolsa y Valores de este país (U.S. Securities and Exchange Commission) (Brachmann, 2020). El contenido de este informe no está regulado y solo en las Reglas Federales de Evidencia (Federal Rules of Evidence), aprobadas por el gobierno en 1972, se indica que las pruebas de los peritos deben basarse en hechos y datos suficientes, y haberse obtenido de acuerdo con una metodología y principios fiables (FRE 702, 2013). No obstante, la estructura típica de un accounting forensic report suele ser la siguiente (Hovland Forensic \& Financial, 2020):

- Indicación de la información recibida (Indication of Information Received). Se trata de un resumen de la información a la que ha 171 tenido acceso el perito para llevar a cabo la investigación.

- Antecedentes (Background of the Case). En este apartado se aporta un resumen del procedimiento judicial (entre un párrafo y varias páginas) y las razones por las que se lleva a cabo la investigación.

- Elementos que se investigan (Items Investigated). Son la parte central del informe en la que se analizan detenidamente todos los elementos que se investigan.

- Conclusiones (Conclusions). En esta parte se indica la cantidad que se sospecha que conforma el fraude.

- Anexos (Exhibits). Son los documentos que prueban los datos que se analizan en la parte de los elementos que se investigan.

En la tabla 1 se muestra de forma comparada las partes del informe en ambos países (en negrita las que consideramos por la información teórica aportada que coincidirían):

Tabla 1. Partes del informe pericial en España y Estados Unidos. Fuente: Elaboración propia

\begin{tabular}{cc}
\hline España & Estados Unidos \\
\hline Identificación y manifestaciones legales & Indication of Information Received \\
\hline $\begin{array}{c}\text { Objeto del informe } \\
\text { Documentación utilizada } \\
\text { Párrafo de reserva de información } \\
\text { Antecedentes y contexto de la situación }\end{array}$ & Background of the Case \\
\hline Documentación examinada & Items Investigated \\
\hline Conclusiones & Conclusions \\
\hline Documentos anexos & Exhibits \\
\hline
\end{tabular}




\subsection{El concepto de género textual: los textos híbridos de contenido jurídico-económico}

En las últimas décadas, el concepto de género textual ha sido el centro de interés de numerosos estudios de investigación en el ámbito de la traducción (Pajares y Alcalde, 2017). De hecho, son numerosos los investigadores que lo utilizan en sus trabajos, sobre todo en traducción jurídica (Borja, 2005; Monzó, 2002, 2005). Tal y como lo defendiera Gamero en 2001, consideramos que utilizar el concepto de género textual es de gran relevancia y utilidad en el ámbito de la traducción, ya que permite definir sus características, lo que conlleva una mejor comprensión del mismo y, por ende, una mejor aplicación de las técnicas necesarias para su traducción. No obstante, no siempre es posible categorizar un género textual de acuerdo con las clasificaciones tradicionales de la traducción, criticadas por Mayoral y Díaz (2011) por no ser de utilidad, ya que no siempre el contenido de un texto es puramente de un campo temático determinado. A este respecto, Mayoral (2002) señalaba como ejemplo que no siempre se pueden separar materias como la traducción económica y la jurídica. En efecto, y tal y como ocurre en el caso de los informes periciales económicos, en los que se presenta de forma híbrida información de índole económico y jurídico, Mateo (2014) señala que los textos que se utilizan para realizar transacciones comerciales tienen efectos jurídicos y por tanto combinan tanto información jurídica como económica. Del mismo modo, Borja (2000 y 2013) señala la confluencia de estos dos campos, algo de lo que son conscientes los traductores que trabajan en estos ámbitos. Por tanto, consideramos un informe pericial económico un texto híbrido con contenido tanto jurídico como económico y que por tanto podrá reunir características de ambos campos, tal y como observaremos en el apartado de análisis tras el estudio de nuestro corpus.

\subsection{Uso de corpus en traducción jurídico- económica}

Cabe señalar que desde mediados del siglo $\mathrm{xx}$ numerosos estudios se han llevado a cabo mediante el uso de corpus, ya que «se conforma como un nuevo paradigma de investigación, más flexible y adaptable, que permite afrontar los retos actuales desde una multiplicidad de perspectivas» (Corpas Pastor, 2008: 49). En este sentido cabe distinguir entre el corpus monolingüe, que permite analizar los rasgos distintivos de textos no traducidos como pueden ser los del discurso o género, y el corpus bilingüe, que permite realizar estudios contrastivos de textos entre dos lenguas (Biel, 2014). No obstante, su uso en traducción jurídica y económica en la combinación lingüística de nuestro estudio (inglés-español) no está tan extendido como en otros ámbitos. Algunos ejemplos recientes de uso de esta metodología lo constituyen el estudio realizado por Alcalde y Santamaría (2019) sobre el uso del término compliance, a caballo entre el ámbito jurídico y económico, en diarios de corte generalista y especializado. En esta misma línea encontramos el realizado por Vigier y Sánchez (2017) con el objetivo de comparar términos jurídicos entre el inglés y el español. Para ello, proceden a la alineación de sentencias en inglés y en español dictadas por tribunales de Inglaterra. Desde un ángulo más enfocado a la didáctica, Biel (2017) aporta en su estudio resultados sobre cómo el uso de un corpus comparable en el aula contribuye a aumentar la dimensión comunicativa de los traductores jurídicos y lo defiende desde una doble perspectiva; por un lado, con un objetivo práctico que contribuya a realizar una traducción $y$, por otro, con el objetivo de reflexionar sobre el proceso de trabajo. Finalmente, resulta interesante mencionar el proyecto COMENEGO: Corpus Multilingüe en Economía y Negocios 
(2017). Este proyecto aporta un corpus especializado como recurso tanto para investigadores, traductores en formación y profesionales para cubrir así las necesidades específicas de este ámbito de la traducción.

\section{METODOLOGÍA}

La metodología de nuestro estudio se basa en un análisis de corpus comparable con el objetivo de caracterizar la macroestructura y la microestructura del informe pericial económico en dos lenguas (español e inglés) y en dos países (España y EE. UU). Debido al carácter con- fidencial de estos documentos, hemos limitado el corpus a un total de seis documentos (tres en inglés y tres en español) obtenidos de fuentes abiertas en la web. El corpus se compone de 41 943 palabras en inglés y 117492 en español. A cada uno de ellos se les ha asignado una letra que nos permitirá identificarlos y referirnos a ellos a lo largo del artículo.

A pesar de que no se trate de un corpus de gran amplitud, consideramos que la muestra nos permitirá realizar una primera caracterización y definición de este tipo de documentos. Con el objetivo de presentar la información de

Tabla 2. Informes periciales analizados. Fuente: Elaboración propia

\begin{tabular}{|c|c|c|c|c|c|}
\hline \multicolumn{6}{|c|}{ INFORMES PERICIALES ANALIZADOS } \\
\hline & INGLÉS & & & ESPAÑNOL & \\
\hline $\mathbf{A}$ & $\begin{array}{l}\text { Bernalillo Public Schools - } \\
\text { Forensic Audit Consulting } \\
\text { Procedures }{ }^{1}\end{array}$ & $\begin{array}{c}29365 \\
\text { Palabras }\end{array}$ & D & Mansolivar - Informe Pericial ${ }^{2}$ & 110003 palabras \\
\hline B & $\begin{array}{c}\text { Forensic Accountants' } \\
\text { Report }^{3}\end{array}$ & 21159 palabras & $\mathbf{E}$ & $\begin{array}{l}\text { VPF y Galdon Software - Informe } \\
\text { Pericial }^{4}\end{array}$ & 3875 palabras \\
\hline C & Forensic Audit ${ }^{5}$ & 6146 palabras & $\mathbf{F}$ & $\begin{array}{c}\text { Registro de Auditores Judiciales y } \\
\text { Forenses - Emisión de informes } \\
\text { periciales }^{6}\end{array}$ & 3614 palabras \\
\hline & TOTALES & 56670 palabras & & TOTALES & $\begin{array}{c}117492 \\
\text { palabras }\end{array}$ \\
\hline
\end{tabular}

I https://media.krqe.com/nxs-krqetv-media-us-east-1/document_dev/2018/04/04/7007_Bernalillo_Public_Schools_ Consulting_Procedures_Sept_30_2016_1522899849043_39160404_ver1.0.pdf

2 https://d500.epimg.net/descargables/2015/03/30/5fb73fd254ac2cf6a6ec721f88d483ec.pdf?rel=mas

3 https://www.nigrini.com/EndofChapter/Rhodes_ForensicReport.pdf

4 https://csuelo.galdon.com/ejemplo_informe_pericial.pdf

5 https://www.sampletemplates.com/business-templates/report/forensic-audit-report-samples-templates.html

6 https://www.diarioabierto.es/wp-content/uploads/2019/01/cuaderno-pr\%C3\%A1ctico-raj1.pdf 
174 la manera más clara y concisa posible, hemos adaptado la metodología de análisis aplicada por Alcalde y Santamaría (2019) en un estudio previo y que permite analizar tanto elementos verbales como no verbales, tal y como iremos mostrando en las diferentes secciones. Esta misma metodología ha sido aplicada en estudios previos por parte de otros autores (Borrueco Rosa, 2005, 2006; Ponce Márquez, 2008). Del mismo modo, se ha utilizado la herramienta de gestión de corpus Sketch Engine para analizar en mayor detalle la frecuencia de palabras de este tipo de informes, así como los elementos verbales más característicos. Se trata de un software de gestión de corpus y análisis de texto en línea que ofrece a sus usuarios la posibilidad de llevar a cabo múltiples funciones para el análisis lingüístico de corpus. Una vez seleccionados los textos, hemos creado dos corpus diferentes. Por un lado, el corpus con los textos en español y, por otro, el corpus con los textos en inglés. La herramienta permite hacer búsquedas de colocaciones y combinaciones de palabras (Word Sketch), así como de expresiones multipalabra, acceder a un tesauro, hacer comparaciones de palabras a través de colocaciones, y realizar extracciones terminológicas. En el caso del análisis que nos ocupa, destacamos el uso de este software, puesto que nos ha facilitado la visualización de ejemplos de los elementos verbales del estudio en su contexto (a través de la función denominada Concordancia), así como acceder a un listado con los términos clave de los informes periciales. Se trata, como se puede observar, de una herramienta muy completa para el análisis lingüístico y para la compilación de corpus. Otra de las funciones destacables es la posibilidad de recurrir a corpus creados por otros usuarios. En el momento de elaboración de este artículo había disponibles 520 corpus en 95 lenguas diferentes.

\section{ANÁLISIS DE RESULTADOS}

En este apartado aportaremos una visión general de la estructura, del léxico y de las expresiones más empleadas en este tipo de documentos en inglés y en español.

\subsection{Elementos no verbales}

Este primer apartado se centra en cuestiones relacionadas con la macroestructura del texto. Esto nos permitirá determinar si la estructura que se ha descrito en el marco teórico de este artículo se cumple o si, por el contrario, estos documentos carecen de un esquema común en ambos idiomas. Los elementos que se estudiarán en este apartado, tanto en inglés como en español, serán tres: (1) índice y capítulos, (2) tipos y estilos de letra y (3) tablas y gráficas. Al finalizar la caracterización de estos elementos se incluirá un resumen con los aspectos más significativos.

\subsubsection{Español}

\subsubsection{1. Índice y capítulos}

Tal y como se apuntaba en el apartado 2.1. de este artículo, existe una estructura típica de los informes periciales contables (Bermúdez, 2019) que, por lo general, se divide en cinco grandes apartados: (1) identificación y manifestaciones legales, (2) antecedentes y contexto de la situación, (3) documentación examinada, (4) conclusiones y (5) documentos anexos. Tras el análisis de los informes periciales en español podemos afirmar que, aunque sí existen denominadores comunes entre los tres modelos, cada emisor opta por incluir capítulos diferentes que responden a las características específicas de cada procedimiento judicial y a la necesidad de extenderse en la explicación de los datos. A continuación, se incluye una tabla en la que se pueden leer los capítulos incluidos en cada informe estudiado: 
Tabla 3. Índice de capítulos de los informes periciales en español. Fuente: Elaboración propia

\begin{tabular}{|c|c|c|c|}
\hline & $\mathrm{D}$ & E & $\mathrm{F}$ \\
\hline 1 & $\begin{array}{l}\text { Introducción } \\
\text { Alcance } \\
\text { Perito que suscribe } \\
\text { Encargo }\end{array}$ & $\begin{array}{c}\text { Introducción } \\
\text { Perito que suscribe } \\
\text { Carácter del informe } \\
\text { Consideraciones previas }\end{array}$ & $\begin{array}{l}\text { Identificación del documento, } \\
\text { del perito, del solicitante y mani- } \\
\text { festaciones legales. }\end{array}$ \\
\hline 2 & Resumen ejecutivo & $\begin{array}{c}\text { Objetivos } \\
\text { Resumen ejecutivo }\end{array}$ & Objeto del dictamen \\
\hline 3 & Contenido y estructura del informe & Antecedentes & $\begin{array}{l}\text { Antecedentes y contexto de la } \\
\text { situación }\end{array}$ \\
\hline 4 & Normativa & Desarrollo y metodología & Documentación examinada \\
\hline 5 & Situación económico-financiera (1) & Resultados & $\begin{array}{l}\text { Párrafo de reserva de informa- } \\
\text { ción }\end{array}$ \\
\hline 6 & Configuración del grupo & Conclusiones & Procedimientos y metodología \\
\hline 7 & Situación económico-financiera (2) & Anexos & Conclusiones \\
\hline 8 & Salida a bolsa & & $\begin{array}{c}\text { Delimitación de responsabili- } \\
\text { dades }\end{array}$ \\
\hline 9 & Situación económico-financiera (3) & & \\
\hline 10 & Reformulación de las cuentas & & \\
\hline 11 & Proceso de recapitalización & & \\
\hline 12 & Ajuste definitivo & & \\
\hline 13 & $\begin{array}{l}\text { La auditoría de la información } \\
\text { financiera }\end{array}$ & & \\
\hline 14 & Imagen fiel & & \\
\hline 15 & $\begin{array}{l}\text { Fecha de conclusión y entrega del } \\
\text { informe }\end{array}$ & & \\
\hline 16 & Anexos & & \\
\hline
\end{tabular}

Los capítulos de los informes recogidos en la tabla anterior muestran que el informe D es mucho más extenso y completo que los informes $\mathrm{E} y$ $\mathrm{F}$, principalmente por el caso que trata y la profundización que se le quiere dar a cada uno de los apartados. Las celdas sombreadas de la Tabla 3 muestran aquellas secciones que comparten los tres informes. Por tanto, teniendo en cuenta la información recabada en el marco teórico y la analizada en estos tres informes, podemos señalar que no existe un único modelo de informe pericial contable en español, ya que este puede variar dependiendo del contexto, del proceso particular o de la situación de la empresa para la que se haga el informe. Sin embargo, partiendo de los datos analizados, podemos determinar de forma preliminar que la estructura en español se caracteriza por tener los siguientes apartados: 
176 Tabla 4. Capítulos que debe incluir el informe pericial económico en español. Fuente: Elaboración propia

\begin{tabular}{cc}
\hline & $\begin{array}{c}\text { CAPÍTULOS DEL INFORME } \\
\text { PERICIAL ECONÓMICO }\end{array}$ \\
\hline 1 & $\begin{array}{c}\text { Introducción } \\
\text { Información sobre el perito } \\
\text { Información sobre el encargo }\end{array}$ \\
\hline 2 & Manifestaciones legales y normativa \\
\hline 3 & Objetivos \\
\hline 4 & Antecedentes \\
\hline 5 & Desarrollo y metodología \\
\hline 7 & Conclusiones \\
\hline 7
\end{tabular}

El informe D no incluye un capítulo específico dedicado al desarrollo y la metodología, porque este ha sido dividido en diez secciones diferentes (de la 5 a la 14), mientras que los informes $\mathrm{E}$ y $\mathrm{F}$ sí que cuentan con una única sección en la que han desarrollado el informe y presentado la metodología que se va a emplear. En definitiva, los informes de este tipo en español pueden incluir capítulos diferentes y no compartir un único índice, pero podemos afirmar que existen ciertos apartados que se incluyen en todos ellos.

\subsubsection{Tipos y estilos de letra}

En cuanto a los tipos y estilos de letra, nos centraremos aquí en el uso que se hace de la negrita, de la cursiva y del subrayado para determinar similitudes y diferencias entre los informes analizados. Para ello, presentaremos en la siguiente tabla (Tabla 5) los elementos a los que los textos recurren en relación con los tres tipos y estilos de letra descritos.

$\mathrm{Al}$ igual que ocurría en el apartado anterior, el uso de los tipos y estilos de letra también dependerá del emisor del informe. Sin embargo, hay algunos aspectos que sí comparten al menos dos de los informes (sombreados en la Tabla 5) y que muestran que la negrita se emplea en la gran mayoría de las ocasiones para destacar información y resaltarla del resto de los datos incluidos. Asimismo, creemos que se trata de una manera de que el lector, teniendo en cuenta que son informes extensos, pueda encontrar la información lo más rápido posible. Destaca el uso de la negrita para resaltar los títulos de cada sección, de las oraciones y párrafos más significativos, así como de los nombres propios que se incluyen a lo largo del documento. Por otro lado, se observa que no existe una normativa que rija el uso que se hace de un estilo frente a otro, ya que el informe D, por ejemplo, emplea la cursiva siempre en los reales decretos, mientras que el informe $\mathrm{E}$, recurre a la negrita para mostrar estos mismos elementos. Por último, podemos señalar que hay emisores de dichos informes que prefieren no recurrir a múltiples tipos y estilos de letra diferentes, y optan por un único formato, como es el caso del informe $\mathrm{F}$, el cual únicamente ha recurrido a la cursiva en dos ocasiones a lo largo del documento.

En definitiva, estos informes presentan un tipo de letra claro y de fácil lectura, además de ir acompañados, en ocasiones, de ciertos estilos de letra que permiten resaltar la información y poder buscarla rápidamente. Estos elementos cumplen su función principal: subrayar lo más importante de tan extenso documento.

\subsubsection{Tablas y gráficos}

Dos de los tres informes (D y E) incluyen varias tablas y gráficos a lo largo del documento. El objetivo de incluir estos elementos está en que facilitan la lectura de los datos incluidos y permiten al lector disponer de una idea general tras un rápido vistazo al informe. Las tablas, cuadros y gráficos se emplean en tres ocasiones: 
Tabla 5. Tipos y estilos de letra. Fuente: Elaboración propia

\begin{tabular}{|c|c|c|c|}
\hline & $\mathrm{D}$ & E & $\mathrm{F}$ \\
\hline \multirow{10}{*}{ Negrita } & Cifras totales & Normativa & \\
\hline & Títulos y subtítulos & Títulos de gráficas & \\
\hline & $\begin{array}{c}\text { Nombres propios de la }(\mathrm{s}) \\
\text { empresa }(\mathrm{s})\end{array}$ & $\begin{array}{l}\text { Nombres propios de la }(\mathrm{s}) \\
\text { empresa }(\mathrm{s})\end{array}$ & \\
\hline & $\begin{array}{l}\text { Parte del contenido de las } \\
\text { tablas }\end{array}$ & $\begin{array}{l}\text { Parte del contenido de las } \\
\text { tablas }\end{array}$ & \\
\hline & Oraciones más importantes & Oraciones más importantes & \\
\hline & Cifras más importantes & Cifras más importantes & \\
\hline & \multirow[t]{4}{*}{ Párrafos más significativos } & Títulos (nivel 3) & \\
\hline & & Cifras totales & \\
\hline & & Operaciones matemáticas & \\
\hline & & Fechas & \\
\hline \multirow{3}{*}{ Cursiva } & \multirow[t]{2}{*}{ Reales decretos y normativas } & Encargo & \multirow[t]{3}{*}{ Nombres propios } \\
\hline & & Motivos & \\
\hline & Sentencias & & \\
\hline \multirow{2}{*}{ Subrayado } & \multirow[t]{2}{*}{ Títulos (nivel 3) } & Cifras totales & \\
\hline & & Operaciones matemáticas & \\
\hline
\end{tabular}

- Para resaltar información (cuadros) (Figura 1):

totalidad de requisitos que contemplarían unos estados financieros consolidados completos preparados de acuerdo con las NIIF-UE y por tanto, deben ser leídas conjuntamente con las cuentas anuales de la matriz BFA.
A julio de 2011 el auditor afirma que emitiría un informe favorable sobre las cuentas semestrales del Grupo Bankia salvo que surgiera algún nuevo aspecto significativo a considerar.

Por otro lado, y en lo que respecta a BFA, el 26 de julio de 2011, en la sesión de la Comisión de Auditoría y Cumplimiento de BFA de esa fecha, se presentó el informe sobre las Cuentas Semestrales de BFA (punto 2 del orden del día), "destacando las principales magnitudes del 


\section{Cuadro 28.- Colocación de acciones de la OPS por tramos}

\begin{tabular}{lll}
\hline Tramo & Número de acciones & \% Inicial de la Oferta \\
\hline Minorista y para Empleados & 494.743 .351 & $60 \%$ \\
\hline Inversores Cualificados & 329.828 .902 & $40 \%$ \\
\hline Total acciones & $\mathbf{8 2 4 . 5 7 2 . 2 5 3}$ & \\
Green shoe & 82.457 .226 & \\
\hline Total & $\mathbf{9 0 7 . 0 2 9 . 4 7 9}$ & \\
\hline
\end{tabular}

FIGURA 2. Uso de tablas para incluir cifras (Fuente: Informe D)

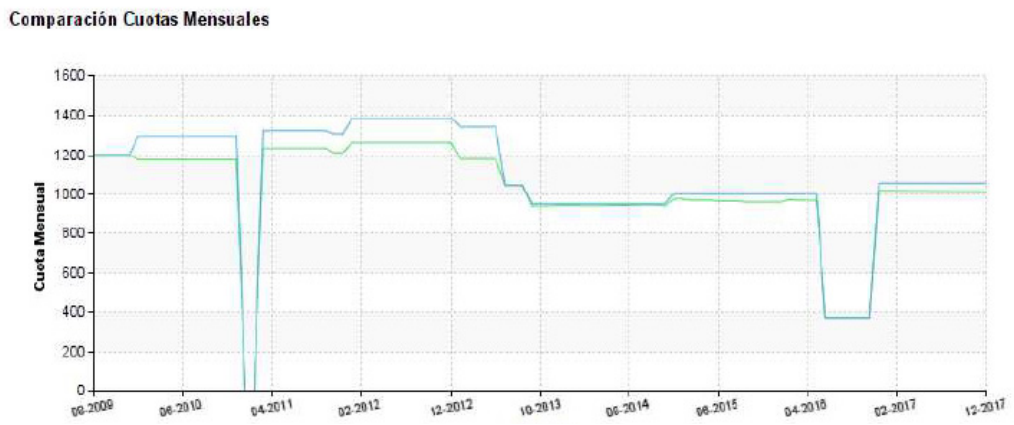

FIGURA 3. Uso de gráficas para mostrar tendencias (Fuente: Informe E)

- Para incluir cifras (tablas) (Figura 2).

- Para mostrar tendencias y situaciones económico-financiera (gráficas) (Figura 3).

Por último, el informe F no incluye ninguno de los recursos descritos en este apartado. De nuevo, esto viene a subrayar la falta de consenso que existe en cuanto a la redacción de este tipo de informes económico-jurídicos y a la inclusión, en este caso, de recursos como tablas, cuadros y gráficos, cuya inclusión dependerá de los objetivos que se persigan.

\subsubsection{Inglés}

A continuación, realizaremos el mismo tipo de análisis, pero, esta vez, de los informes periciales redactados en inglés que conforman nuestro corpus.

\subsubsection{1. Índice y capítulos}

Estos tres documentos, al igual que ocurría en español, no siguen un mismo patrón ni modelo a la hora de redactarlos.

Como muestra la Tabla 6 (celdas sombreadas), los informes en inglés analizados comparten uno 
Tabla 6. Índice de capítulos de los informes periciales contables en inglés. Fuente: Elaboración propia

\begin{tabular}{|c|c|c|c|}
\hline & $\mathbf{A}$ & B & C \\
\hline 1 & $\begin{array}{l}\text { Report of Independent Accoun- } \\
\text { tants }\end{array}$ & Background & Authority Letter \\
\hline 2 & Executive Summary & Executive Summary & Executive Summary \\
\hline 3 & Consulting Procedures & Unique Facts \& Circumstances & $\begin{array}{c}\text { Introduction } \\
\text { Background } \\
\text { Scope and methodology } \\
\text { Requirements }\end{array}$ \\
\hline 4 & Detailed Procedures Performed & Investor Funds Flow & $\begin{array}{l}\text { Audit Findings and Recommen- } \\
\text { dations }\end{array}$ \\
\hline 5 & Corrective Actions to date & $\begin{array}{l}\text { Consolidated Financial State- } \\
\text { ments }\end{array}$ & Exhibit A \\
\hline 6 & Exit conference \& Disclaimer & $\begin{array}{l}\text { Forensic Account Analysis and } \\
\text { Testing }\end{array}$ & Agency Comments \\
\hline 7 & \multirow[t]{2}{*}{ Exhibits } & Findings and Observation & \multirow[t]{2}{*}{ State Controller's Comments } \\
\hline 8 & & $\begin{array}{c}\text { Current Financial Position of the } \\
\text { Estate }\end{array}$ & \\
\hline
\end{tabular}

de los capítulos: executive summary. Existen otras secciones que solo comparten dos de los informes como, por ejemplo, el apartado exhibits (A y C), el de audit findings and recommendations/observation (B y C) o el de background (B y C). Al igual que ocurría en español, dependiendo de la extensión del documento y, principalmente, de la temática, los emisores pueden necesitar incluir apartados más específicos. De nuevo, estos resultados vienen a resaltar que no existe un modelo único y que no se siguen las mismas partes que se indican en la teoría (Hovland Forensic \& Financial, 2020) al no contar con legislación específica al respecto. No obstante, es necesario señalar que, a pesar de las diferencias en la estructura, los informes incluyen información muy parecida. Teniendo en cuenta los documentos analizados y el marco teórico estudiado en el apartado 2.1 de este artículo, a continuación, se muestra una estructura que permitiría caracterizar de forma preliminar un informe pericial en inglés (Tabla 7).

Tabla 7. Estructura preliminar de un informe pericial contable en inglés. Fuente: Elaboración propia

\begin{tabular}{ll}
\hline & $\begin{array}{l}\text { CAPÍTULOS DEL INFORME PERICIAL } \\
\text { ECONÓMICO }\end{array}$ \\
\hline 1 & Executive Summary \\
\hline 2 & $\begin{array}{l}\text { Introduction } \\
\text { Background } \\
\text { Objectives } \\
\text { Scope } \\
\text { Methodology }\end{array}$ \\
\hline 3 & Items investigated \\
\hline 4 & Audit Findings and Recommendations \\
\hline 5 & Annex/Exhibits \\
\hline
\end{tabular}


Tabla 8. Tipos y estilos de letra. Fuente: Elaboración propia

\begin{tabular}{|c|c|c|c|}
\hline & A & B & C \\
\hline \multirow{4}{*}{ Negrita } & Títulos & Títulos & Títulos \\
\hline & Cifras totales & \multirow[t]{3}{*}{ Información reseñable } & Cifras totales \\
\hline & Títulos tablas & & Títulos tablas \\
\hline & Información reseñable & & \\
\hline Cursiva & Citas textuales & Información reseñable & Títulos \\
\hline \multirow{3}{*}{ Subrayado } & Enumeraciones & Títulos & \multirow[t]{3}{*}{ Títulos } \\
\hline & Normativa & Información reseñable & \\
\hline & Criterios & & \\
\hline
\end{tabular}

\subsubsection{Tipos y estilos de letra}

El resumen de los tipos y estilos de letra analizados en los textos en inglés se muestra en la Tabla 8.

De nuevo, destaca la ausencia de un criterio fijo a la hora de emplear unos estilos de letra frente a otros. Será el emisor en todo momento en el que elija, de acuerdo con su criterio propio, qué estilos emplear y cuándo emplearlos.

\subsubsection{Tablas y gráficos}

Las tablas se incluyen en los tres informes analizados. Sin embargo, el informe B, además de las tablas, también recurre a gráficos para presentar la información de una forma más clara y visual para el lector. Las ocasiones en las que se recurre a estos elementos son las siguientes:

- Para incluir cifras (tablas) (Figura 4).

- Para incluir resúmenes de la información descrita en el cuerpo del texto (tablas).

- Para resaltar información e incluir notas (cuadros).

- Para incluir tendencias (gráficos) (Figura 5).
Los emisores de estos tres informes hacen uso de este recurso para facilitar la lectura y que el lector pueda ir directamente a la información que más le interese en cada momento. El uso que hacen de estos recursos no es el mismo, por lo que podríamos decir que, de nuevo, estamos ante una falta de consenso en cuanto al uso de tablas, gráficos y cuadros en los informes periciales contables en inglés. Sin embargo, sí podemos subrayar que el uso de las tablas es común en los tres informes y que se emplean, en la gran mayoría de las ocasiones, para incluir resúmenes de cifras.

\subsubsection{Resumen de elementos no verbales}

Como se ha podido comprobar en los cuatro apartados dedicados a los elementos no verbales de los informes en español y en inglés, no podemos afirmar que haya un modelo y una estructura fija que se respete en todos ellos. En lo que respecta a los capítulos, existen secciones comunes e indispensables, pero, en cuanto al contenido, la forma varía y depende de las empresas que se 


\begin{tabular}{|l|c|c|c|c|c|}
\hline & \multicolumn{1}{|c|}{$\begin{array}{c}\text { Annual } \\
\text { Pension } \\
\text { Based on } \\
\text { Employee/Age }\end{array}$} & $\begin{array}{c}\text { Base } \\
\text { Salary }\end{array}$ & $\begin{array}{c}\text { Pension } \\
\text { Bnrichments* }\end{array}$ & $\begin{array}{c}\text { Total } \\
\text { Annual } \\
\text { Pension* }\end{array}$ & $\begin{array}{c}\text { Total } \\
\text { Lifetime } \\
\text { Pension } \\
\text { Enrichments* }\end{array}$ \\
\hline $\begin{array}{l}\text { Assistant } \\
\text { Supervisor/58** }\end{array}$ & $\$ 75,920$ & $\$ 47,713$ & S61,716 & $\$ 109,429$ & $\$ 1,542,900$ \\
\hline $\begin{array}{l}\text { Assistant } \\
\text { Supervisor/57*** }\end{array}$ & 75,920 & 41,616 & 32,888 & 74,504 & 822,200 \\
\hline Signalman/56** & 64,106 & 38,066 & 23,697 & 61,763 & 592,425 \\
\hline Signalman/59*** & 64,106 & 35,280 & 28,930 & 64,210 & 723,250 \\
\hline Signalman/49 & 67,954 & 31,041 & 36,115 & 67,156 & 902,875 \\
\hline $\begin{array}{l}\text { Assistant } \\
\text { Foreman/60** }\end{array}$ & 67,954 & 24,120 & 38,752 & 62,872 & 968,800 \\
\hline
\end{tabular}

Figura 4. Uso de tablas para incluir cifras. FUENTE: Informe $C$

\section{Fnancing Cash Flow}

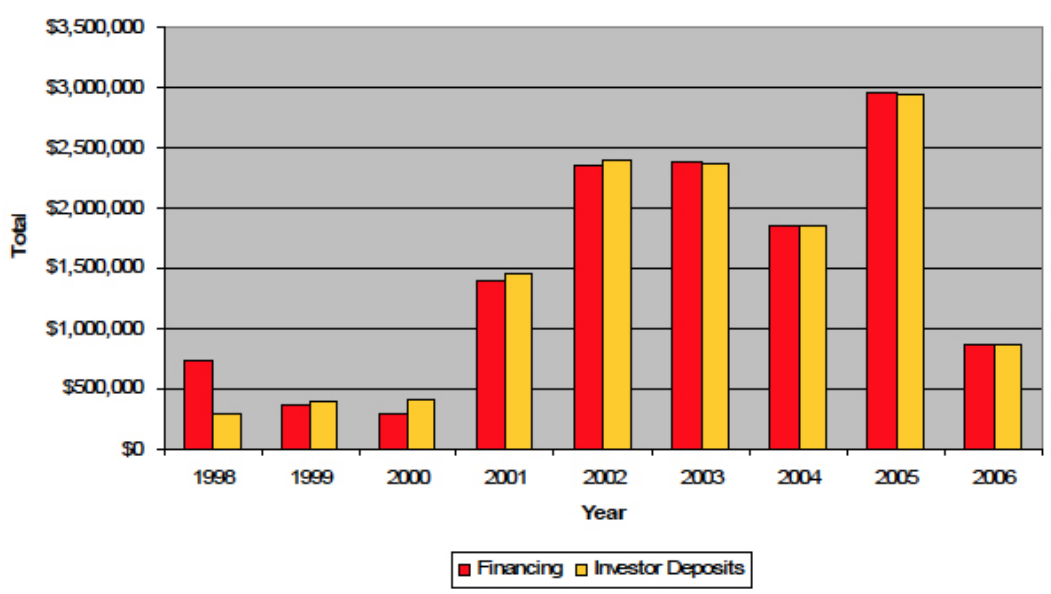

Figura 5. Uso de gráficos para mostrar tendencias. FUENTE: Informe B

encargan de la redacción de dichos informes. Teniendo en cuenta el análisis realizado, esta sería de forma preliminar la estructura que caracterizaría a los informes periciales contables que se usan en el ámbito judicial en inglés y en español (Tabla 9).
En lo que respecta a los tipos y estilos de letra tampoco existe un criterio común, pero podríamos establecer de forma preliminar las siguientes características (Tabla 10).

En resumen, no existe en lo que respecta a los elementos no verbales de este tipo de documen- 
Tabla 9. Estructura preliminar de los informes periciales contables en español y en inglés.

Fuente: Elaboración propia

\begin{tabular}{ccc}
\hline & ESPAÑOL & INGLÉS \\
\hline 1 & $\begin{array}{c}\text { Introducción } \\
\text { Información sobre el perito } \\
\text { Información sobre el encargo }\end{array}$ & $\begin{array}{c}\text { Introduction } \\
\text { Background } \\
\text { Objectives } \\
\text { Scope }\end{array}$ \\
\cline { 1 - 2 } 2 & Objetivos & \\
\hline 3 & Manifestaciones legales y normativa & Executive Summary \\
\hline 5 & Antecedentes & Items investigated \\
\hline 6 & Desarrollo y metodología & Audit Findings and Recommendations \\
\hline 7 & Conclusiones & Annex/Exhibits \\
\hline
\end{tabular}

Tabla 10. Tipos y estilos de letra de los informes periciales contables en español y en inglés.

Fuente: Elaboración propia

\begin{tabular}{|c|c|c|}
\hline & ESPAÑOL & INGLÉS \\
\hline \multirow{6}{*}{ Negrita } & $\begin{array}{l}\text { Títulos y subtítulos } \\
\text { Títulos tablas }\end{array}$ & $\begin{array}{l}\text { Títulos y subtítulos } \\
\text { Títulos tablas }\end{array}$ \\
\hline & Cifras totales & Cifras totales \\
\hline & Información reseñable & \multirow[t]{4}{*}{ Información reseñable } \\
\hline & Nombres propios & \\
\hline & Fechas & \\
\hline & Normativa & \\
\hline \multirow{4}{*}{ Cursiva } & Normativa & Citas textuales \\
\hline & Encargos & Información reseñable \\
\hline & Motivos & \multirow[t]{2}{*}{ Títulos } \\
\hline & Nombres propios & \\
\hline \multirow{5}{*}{ Subrayado } & Títulos & Enumeraciones \\
\hline & Cifras totales & Normativa \\
\hline & \multirow{3}{*}{$\begin{array}{c}\text { Ecuaciones } \\
\text { Operaciones matemáticas }\end{array}$} & Criterios \\
\hline & & Títulos \\
\hline & & Información reseñable \\
\hline
\end{tabular}


tos, un criterio común, ya que estos se utilizan de manera diferente dependiendo del emisor y características específicas de cada informe.

\subsection{Elementos verbales}

En este apartado presentamos una aproximación de las características verbales del tipo de documento que nos ocupa. Analizaremos, por un lado, el plano morfosintáctico y, por otro, el plano léxico-semántico.

\subsubsection{Plano morfosintáctico}

En este apartado se analizan las características correspondientes a la voz del verbo y a los tiempos verbales en ambos idiomas. Por un lado, el número de verbos en el corpus en español asciende a 12 362, de los cuales 345 corresponden a verbos en voz pasiva. Por otro lado, en inglés se incluyen 5068 verbos, de los que 397 emplean la voz pasiva. Si tomamos los porcentajes de uso de la pasiva en inglés (7,8 \%), esta se emplea con mayor frecuencia en comparación con el uso que se hace de ella en los informes periciales en español (2,79\%). En la Tabla 11 se incluyen dos ejemplos de su uso.
En cuanto a los tiempos verbales, en ambos idiomas el tiempo verbal más empleado es el presente de indicativo, seguido por el pasado simple en inglés y el pretérito perfecto compuesto en español. Esto se debe, principalmente, a que estos informes resumen las acciones llevadas a cabo por los emisores con anterioridad a la redacción del documento y a que describen lo que ocurre actualmente en la empresa, es decir, presentan una realidad, así como la situación económica actual (Tabla 12).

En tercer lugar, ambos idiomas recurren con mayor frecuencia al futuro imperfecto, lo cual se debe a que otra de las funciones de estos informes es determinar acciones futuras que la empresa debe llevar a cabo para solventar los problemas detectados durante la fase de análisis (Tabla 13).

En cuarto lugar, el condicional simple, tanto en inglés como en español, está presente, aunque en un porcentaje menor. Su uso se limita a las ocasiones en las que el emisor incluye las acciones que se deberían llevar a cabo, a modo de consejo (Tabla 14).

En la tabla 15 se incluye un resumen de las cifras obtenidas durante el análisis.

Tabla 11. La voz del verbo en los informes periciales contables en inglés y en español. Fuente: Elaboración propia

\begin{tabular}{cll}
\hline \multicolumn{1}{c}{ Voz del verbo } & \multicolumn{1}{c}{ Español } \\
\hline \multicolumn{1}{c}{$\begin{array}{l}\text { In one instance, supervisors and employees were allocating } \\
\text { Hours of Service costs to a capital project that was being } \\
\text { completed by a third-party contractor. }\end{array}$} & $\begin{array}{l}\text { Al objeto de poder elaborar el presente informe me } \\
\text { han sido facilitados los documentos que se rela- } \\
\text { cionan en el Anexo 1. 1.2. Experiencia profesional y } \\
\text { cualificación del perito. }\end{array}$ \\
\hline $2 \quad \begin{array}{l}\text { This consulting engagement was conducted in ac- } \\
\text { cordance with the Audit Act 12-6-6 NMSA 1978, 2.2.2 } \\
\text { NMAC Requirements for Contracting and Conducting. }\end{array}$ & $\begin{array}{l}\text { La evidencia, posteriormente, debe ser cuantificada } \\
\text { para poder concluir que tenía importancia signi- } \\
\text { ficativa. El estudio de las partidas controvertidas } \\
\text { requiere }\end{array}$ \\
\hline
\end{tabular}


184 Tabla 12. Ejemplos de tiempos verbales en presente y en pasado (inglés y español). Fuente: Elaboración propia

Formas verbales en presente y en pasado

\section{Inglés}

It delivers the succinct elements of our findings, with supporting details contained in the pertinent attached exhibits.

During our review of internal controls surrounding the

District's risk assessment process, we

noted that adequate mechanisms are not in place to iden-

tify risks applicable to the District.

\section{Español}

En la realización de este trabajo he analizado la documentación que se detalla a continuación, que considero suficiente para concluir sobre el objeto de este dictamen.

Se han producido las siguientes bonificaciones en el plazo del préstamo hipotecario, dichas bonificaciones se restan de la suma del tipo de referencia más el margen neto.

Tabla 13. Ejemplos de tiempos verbales en futuro (inglés y español). Fuente: Elaboración propia

\begin{tabular}{|c|c|}
\hline \multicolumn{2}{|c|}{ Formas verbales en futuro } \\
\hline Inglés & Español \\
\hline $\begin{array}{l}\text { The Finance Department will conduct on-going financial } \\
\text { trainings with all directors, principals and bookkeepers. }\end{array}$ & Nota: Para el cálculo de intereses se tomarán 365 días. \\
\hline \multicolumn{2}{|c|}{ Tabla 14. Ejemplos de tiempos verbales condicionales (inglés y español). Fuente: Elaboración propia } \\
\hline \multicolumn{2}{|c|}{ Formas verbales condicionales } \\
\hline Inglés & Español \\
\hline $\begin{array}{l}\text { If there was not a report cut-off date for the cash activity, } \\
\text { we would be continuously updating the report through } \\
\text { publication to account for the subsequent receipts and } \\
\text { disbursements. }\end{array}$ & $\begin{array}{l}\text { Las pruebas de resistencia miden la capacidad de las } \\
\text { entidades de absorber fuertes deterioros en el valor de sus } \\
\text { activos, ante escenarios macroeconómicos muy adversos } \\
\text { e improbables, que reducirían, a su vez fuertemente la } \\
\text { solvencia de las mismas. }\end{array}$ \\
\hline
\end{tabular}

A nivel sintáctico conviene igualmente destacar que el texto en inglés recurre a oraciones largas pero sencillas. No obstante, aunque los informes en español también contienen oraciones extensas, estas son de mayor complejidad.

\subsubsection{Plano léxico-semántico}

En este apartado, se analizarán los pronombres personales, determinantes posesivos y unida- des terminológicas clave detectadas durante el análisis y que destacan por su presencia en estos documentos.

En primer lugar, estudiamos el uso que se hace en inglés de la primera persona del plural (171 ocasiones) para incluir en el texto al emisor del informe. Su uso, en cambio, no es tan frecuente en español (60 ocasiones). A diferencia de los pronombres personales, destaca en español el uso que se hace del determinante posesivo 
Tabla 15. Tiempos verbales empleados en ambos idiomas y porcentajes de uso. Fuente: Elaboración propia

\begin{tabular}{cccc}
\hline \multicolumn{1}{c}{ Inglés } & \multicolumn{2}{c}{ Español } \\
\hline Tiempo verbal & Porcentaje & Tiempo verbal & Porcentaje \\
\hline Formas verbales en presente & $93,21 \%$ & Formas verbales en presente & $94,89 \%$ \\
\hline Formas verbales en pasado & $3,76 \%$ & Formas verbales en pasado & $3,24 \%$ \\
\hline Formas verbales en futuro & $2,19 \%$ & Formas verbales en futuro & $1,65 \%$ \\
\hline $\begin{array}{c}\text { Formas verbales condicio- } \\
\text { nales }\end{array}$ & $0,84 \%$ & Formas verbales en condicional & $0,22 \%$ \\
\hline
\end{tabular}

Tabla 16. Ejemplos del uso de pronombres personales y determinantes posesivos en inglés y en español. Fuente: Elaboración propia

Pronombres personales y determinantes posesivos

Inglés

Employees select their work shifts within the parameters of the collective bargaining agreements and we recognize that management cannot arbitrarily move workers to different shifts.

The District remains responsible for their accounting records, activity funds, fraud prevention and detection, and for maintaining effective internal

de primera persona de singular (31 ocasiones) y de plural (83). El determinante posesivo en singular en inglés únicamente aparece en tres ocasiones, mientras que el plural se incluye en el texto 103 veces (Tabla 16).

Al tratarse de un texto objetivo que incluye cifras y datos analizados, el emisor toma parte activa en la redacción del documento y eso se observa en el uso que se hace tanto en inglés como en español de la primera persona del singular y del plural. Destaca especialmente la personalización que toma el texto en español con el uso de los determinantes posesivos de primera persona. En la tabla 17 se muestra un resumen de los datos del análisis en la que se incluye el porcen- taje, del total de palabras, en el que se emplean estos elementos verbales.

En segundo lugar, nos centraremos en aquellas unidades terminológicas clave que determinan la especialización del corpus en ambos idiomas. Los 20 términos clave (sustantivos, adjetivos y verbos) más frecuentes se presentan en la tabla 18.

Como se puede observar en esta lista, el tipo de términos que se detectan con más frecuencia proceden del ámbito jurídico y económico. Sin embargo, no podemos incluir estos términos de forma independiente en un único ámbito de especialización, puesto que una de las características de los informes periciales contables que se usan en el ámbito judicial en ambos idiomas 
Tabla 17. Pronombres personales y determinantes posesivos en inglés y en español. Fuente: Elaboración propia

\begin{tabular}{cccc}
\hline \multicolumn{1}{c}{ Inglés } & \multicolumn{2}{c}{ Español } \\
\hline Pronombres personales & Porcentaje & Pronombres personales & Porcentaje \\
\hline Primera persona (sg./pl.) & $0,33 \%$ & Primera persona (sg./pl.) & $0,05 \%$ \\
\hline Tercera persona (pl.) & $0,17 \%$ & Tercera persona (pl.) & $0,01 \%$ \\
\hline Determinantes posesivos & Porcentaje & Determinantes posesivos & Porcentaje \\
\hline Primera persona (sg./pl.) & $0,19 \%$ & Primera persona (sg./pl.) & $0,09 \%$ \\
\hline Tercera persona (sg./pl.) & $0,18 \%$ & Tercera persona (sg./pl.) & $0,76 \%$ \\
\hline
\end{tabular}

Tabla 18. Palabras clave más frecuentes en español y en inglés. Fuente: Fuente: Elaboración propia

\begin{tabular}{|c|c|}
\hline ESPAÑOL & INGLÉS \\
\hline Diligencias & Forensic \\
\hline Pericial & Entities \\
\hline Perito & Findings \\
\hline Cajas & Procedures \\
\hline Reformulación & Accountants \\
\hline Mutualización & Receiver \\
\hline Solvencia & Audit \\
\hline Subestándar & Digits \\
\hline Amortizativo & Receipts \\
\hline Auditor & Corrective \\
\hline Morosidad & Liabilities \\
\hline Computable & Receivership \\
\hline Crediticio & Auditor \\
\hline Reclasificación & Disbursement(s) \\
\hline Recapitalización & Statements \\
\hline Acreditado & Consulting \\
\hline Reestimación & Embezzlement \\
\hline Segregación & Withdrawals \\
\hline Reformular & Consolidated \\
\hline Recuperable & Account(s) \\
\hline
\end{tabular}


es que estos pueden considerarse como textos de tipo híbrido. Así se indicaba en la introducción al señalar que en el ámbito en el que se enmarcan estos documentos confluyen tanto aspectos jurídicos como económicos con el objetivo de investigar posibles fraudes en el seno empresarial (Singleton y Singleton, 2010). Asimismo, en el marco teórico recogimos estudios previos en los que se hace referencia a textos que no siempre se pueden situar en un campo temático determinado (Mayoral y Díaz, 2011), ya que no siempre se pueden separar materias como la traducción económica y jurídica (Borja, 2000 y 2013; Mateo, 2014; Mayoral, 2002).

Con el fin de explicar esta situación, se han introducido, por un lado, los términos más frecuentes recogidos en español en la Tabla 18 en el Corpus del Español del Siglo XXI (CORPES). La razón de emplear este recurso es que se trata de un banco de datos de la lengua española en el que se incluye un amplio número de textos (285 000) de diversos ámbitos, almacenados en soporte electrónico. Se observa que 18 de los términos se incluyen dentro del tema política, economía y justicia, uno de ellos no aparece en el listado de concordancias (amortizativo), uno de los términos (segregación) se encuadra dentro del ámbito de las ciencias sociales, creencias y pensamiento, aunque el segundo ámbito de aparición es el que corresponde a la política, economía y justicia.

Por otro lado, hemos realizado la misma operación con los términos en inglés y, para ello, se ha recurrido al diccionario Merriam Webster, ya que este recurso ofrece, para cada uno de ellos, el ámbito de especialización. De esta forma, los dos grandes ámbitos a los que pertenecen los términos clave de nuestro corpus son el jurídico y el financiero, lo que vuelve a subrayar el carácter híbrido de los documentos analizados. Además, existen términos que se emplean de manera paralela en ambos ámbitos. A continuación, se in- cluye un listado con los términos y el ámbito de especialización al que pertenecen.

\section{CONCLUSIONES}

No cabe duda de la relevancia que presentan en la actualidad los informes periciales contables en el ámbito judicial, al ser un encargo frecuente debido a la globalización de los mercados. Ante la falta de investigaciones previas que se centren de forma específica en este tipo de texto desde el punto de vista traductológico, consideramos de utilidad realizar un estudio que permitiera contar con una primera aproximación a su caracterización en inglés y español como paso previo a su traducción. Tras una contextualización

Tabla 19. Ámbitos de especialización de las palabras clave más frecuentes del corpus en inglés.

Fuente: Elaboración propia

\begin{tabular}{cc}
\hline ÁMBITO JURÍDICO & $\begin{array}{c}\text { ÁMBITO } \\
\text { FINANCIERo }\end{array}$ \\
\hline Findings & Accountant \\
\hline Receiver & Digits \\
\hline Receipt & Disbursement \\
\hline Embezzle & Consolidated \\
\hline Statement & Consulting \\
\hline Withdrawal \\
\hline Forensic \\
\hline Entities \\
\hline Procedure \\
Account \\
\hline \multicolumn{2}{c}{ Audit } \\
\hline Leceivership \\
\hline Liability \\
\hline
\end{tabular}


188 a nuestro objeto de estudio y un marco teórico en el que revisamos los principales trabajos de la literatura existente del ámbito jurídico-económico y de corpus relacionados con nuestra investigación, detallamos en la metodología cómo se procedió a la realización del corpus a través de la herramienta Sketch Engine. Aunque somos conscientes de las limitaciones que el número de textos que lo conforman plantea para este estudio, consideramos que cumplimos con el objetivo de realizar un primer acercamiento a la caracterización de este tipo de texto en ambos idiomas al haber analizado tanto los elementos de su macroestructura y de su microestructura. Esto supone un recurso de gran fiabilidad y utilidad para aquellos traductores y docentes que trabajen o tengan interés en conocer los informes periciales contables con mayor grado de detalle como paso previo a su traducción.

En relación con los datos obtenidos, podemos señalar que no se detecta un único modelo de informe que presente características convencionalizadas en cuanto a su estructura y detalles de tipo de letra o uso de elementos gráficos. En lo que respecta a los elementos verbales, destaca principalmente el uso de la voz pasiva en inglés y su poca presencia en español. Lo que sí comparten estos informes en los dos idiomas es el uso que hacen de los tiempos verbales, puesto que el presente, el pasado, el futuro y el condicional, respectivamente, son los cuatro tiempos verbales más empleados en ambos. Destaca especialmente el uso de los determinantes posesivos y pronombres personales de primera persona, principalmente del plural para incluir el punto de vista del emisor. Finalmente, las unidades terminológicas más frecuentes detectadas en el análisis dan muestra de la especialización del campo temático en el que se enmarcan y del carácter híbrido de este tipo de textos.
Somos conscientes de las limitaciones que presenta esta investigación teniendo en cuenta la amplitud del ámbito del saber en el que se enmarcan los informes periciales contables. Por ello, pretendemos seguir dándole continuidad ampliando el número de textos de nuestro corpus, así como la procedencia de los textos, para seguir estudiando sus características y detenernos en el análisis de las unidades terminológicas y fraseológicas. Del mismo modo, será de gran utilidad, a partir de estos resultados, realizar un glosario detallado con equivalencias y ejemplos contextualizados que contribuya al proceso de traducción de estos textos.

\section{REFERENCIAS BIBLIOGRÁFICAS}

ACFE (2020): «Report to the Nations. 2020 Global Study on Occupational Fraud and Abuse», $<$ https://acfepublic.s3-us-west-2.amazonaws. com/2020-Report-to-the-Nations.pdf> [consulta: 14-V-2020].

Alcalde, Elena y Santamaría, Alexandra. (2019): «Compliance or cumplimiento normativo? A corpus study with professional and didactic purposes in the Spanish press», Revista de Lingüistica y Lenguas Aplicadas, 14, 19-27.

Alcalde, Elena y Santamaría, Alexandra (2019). Translation and Communication in the Promotion of Business Tourism: Emerging Research and Opportunities, IGI Global.

BALL, Ray (2009): «Market and Political/Regulatory Perspectives on the Recent Accounting Scandals», Journal of Accounting Research, 47/2, 277-323.

BIEL, Lucja (2017): «Enhancing the communicative dimension of legal translation: comparable corpora in the research-informed classroom», The Interpreter and Translator Trainer, 11/4, 316-336.

BIEL, Lucja (2014): Lost in the Eurofog: The Textual Fit of Translated Law, Fráncfort del Meno: Peter Lang.

BorJA, Anabel (2000): «La traducción jurídica: didáctica y aspectos textuales», <http://cvc.cervantes. es/obref/aproximaciones/borja.htm> [consulta: 14-V-2020]. 
BORJA, Anabel (2005): «Organización del conocimiento para la traducción jurídica a través de sistemas expertos basados en el concepto de género textual», en Isabel García Izquierdo (ed.), El género textual y la traducción. Reflexiones teóricas y aplicaciones pedagógicas, Berne: Peter Lang, 37-68.

BorJa, Anabel (2013): «Freelance Translation for Multinational Corporations and Law Firms», en Anabel Borja y Fernando Prieto (eds.), Legal Translation in Context: Professional Issues and Prospects, Berna: Peter Lang, 53-74.

Borrueco Rosa, María de los Ángeles (2005-2006): «El discurso turístico: Estudio lingüístico aplicado a la enseñanza de la traducción», Hieronymus Complutensis, 12, 69-78.

Brachmann, Steve (2020): «Regulations in Forensic Accounting», <https://smallbusiness.chron.com/ regulations-forensic-accounting-25817.html> [consulta: 14-V-2020].

BERMÚDEZ, Isabel (2019): «Cuadernos práctico no 1 . Emisión de informes periciales», https://www.icjce.es/adjuntos/cuaderno-practico-raj1.pdf> [consulta: $14-\mathrm{V}-2020]$.

Cinco Días (2019): «EY halla indicios de fraude en su auditoría forense sobre Dia», <https://cincodias.elpais.com/cincodias/2019/02/07/companias/1549573998_066999.html> [consulta: 14-V2020].

COMENEGO (2017): «Corpus Multilingüe de Economía y Negocios», <https://dti.ua.es/comenego>. [consulta: 14-V-2020].

CORPAS PASTOR, Gloria (2008): Investigar con corpus en traducción: los retos de un nuevo paradigma, Fráncfort del Meno: Peter Lang.

Davis, Charles, Farrell, Ramona y OGilby, Suzanne (2010): «Characteristics and skills of the forensic accountant», <http://www.aicpa.org/InterestAreas/ForensicAndValuation/Resources/PractAidsGuidance/DownloadableDocuments/ForensicAccountingResearchWhitePaper.pdf $>$. [consulta: 14-V-2020].

Deloitte (2019): «Servicios de investigación forense», $<$ https://www2.deloitte.com/cl/es/pages/finance/ solutions/cl-auditoria-forense.html> [consulta: 14-V-2020].
GAMERo, Silvia (2001): La traducción de textos técnicos: Descripción y análisis de textos (alemán-español), Barcelona: Ariel.

Hovland Forensic \& Financial (2020): «Ultimate Guide to Forensic Accounting» <https://hovlandforensic. com/forensic-accounting-the-ultimate-guide/> [consulta: 14-V-2020].

HUber, Dennis (2013): «Forensic Accounting Corporations Codes of Ethics and Standards of Practice: A Comparison», Journal of Forensic \& Investigative Accounting, 6/3, 154-170.

Huber, Dennis (2012): «Is Forensic Accounting in the United States Becoming a Profession?», Journal of Forensic \& Investigative Accounting, 4/1, 255-284.

Mateo, José (2014): «Aspectos organizativos y formativos en la enseñanza universitaria de la traducción de negocios en España», en Daniel Gallego (ed.), Traducción económica: entre profesión, formación y recursos documentales, Zaragoza: Vertere, 57-78.

Mayoral, Roberto y Díaz, Óscar (2011): La traducción especializada y las especialidades de la traducción. Castellón de la Plana: Universitat Jaume I.

Mayoral, Roberto (2002): «¿Cómo se hace la traducción jurídica?», Puentes. Hacia nuevas investigaciones en la mediación intercultural, 2, 9-14, <http:// wdb.ugr.es/ greti/revista-puentes/pub2/02-articulo.pdf> [consulta: 14-V-2020].

Monzó, Esther (2002): La professió del traductor jurídic $i$ jurat. Descripció sociològica de la professió $i$ anàlisi discursiva del transgènere. Tesis doctoral. Castellón: Universidad Jaume I. <http://repositori.uji. es/xmlui/handle/10234/29702> [consulta: 14-V2020].

Monzó, E. (2005): «Reeducación y desculturalización a través de géneros en traducción jurídica, económica y administrativa», en Isabel García Izquierdo (ed.), El género textual y la traducción. Reflexiones teóricas y aplicaciones pedagógicas, Berna: Peter Lang.

Pajares, José Sergio y AlCalde, Elena (2017): «Hybrid texts and their influence on the work carried out by specialized translators», Revista Española de Lingüística Aplicada, 30/2, 514-537.

Pierino Dell'Elce, Quintino (2017): «Algunos aspectos importantes a tener en consideración previo a or- 
190 denar un estudio pericial contable», <http://www. economicas.uba.ar/wp-content/uploads/2017/06/ SimposioXXX_A_02_Dell_elce.pdf> [consulta: 14V-2020].

Ponce Márquez, N. (2006): «Metodología en la clase de traducción: dificultades pragmático-culturales en la traducción de textos turísticos (Ejemplos de traducción español /alemán)», Revista electrónica de estudios filológicos, 12.

Secretaría de Estado de Comercio (2020): «Estados Unidos», <https://www.icex.es/icex/es/ navegacion-principal/todos-nuestros-servicios/ informacion-de-mercados/paises/navegacionprincipal/el-pais/relaciones-bilaterales/index. html?idPais=US $>$ [consulta: $14-\mathrm{V}-2020]$.

Singleton, Tommie W., y Aaron J. Singleton (2006): Fraud Auditing and Forensic Accounting, Nueva York: John Wiley \& Sons.

Stanbury, J., and C. Paley-Menzies (2010): «Forensic Futurama: Why Forensic Accounting Is Evolving», <http://www.aicpa.org/Publications/Newsletters/ AICPACPAInsider/2010/jun28/Pages/ForensicFuturamaWhyForensicAccountingIsEvolving.asp $\mathrm{x}>$ [consulta: 14-V-2020].

Vigier, Francisco Javier y SÁnchez, María del Mar (2017): «Using Parallel Corpora to Study the Translation of Legal System-bound Terms: the Case of Names of English and Spanish Courts», en Gloria Corpas Pastor y Ruslan Mitkov (eds), Computational and Corpus-based Phraseology, Nueva York: Springer, 1-14. 